\title{
Organic food consumption during pregnancy is associated with different consumer profiles, food patterns and intake: the KOALA Birth Cohort Study
}

\author{
Ana Paula Simões-Wüst ${ }^{1,2, *}$, Carolina Moltó-Puigmarti ${ }^{3}$, Martien CJM van Dongen ${ }^{3,4}$, \\ Pieter C Dagnelie ${ }^{3,4}$ and Carel Thiij ${ }^{3}$ \\ ${ }^{1}$ Research Department, Clinic Arlesheim, Arlesheim, Switzerland: ${ }^{2}$ Department of Obstetrics, Perinatal \\ Pharmacology, Zurich University Hospital, Schmelzbergstrasse 12/PF 125, Path G 51 la, 8091 Zurich, Switzerland: \\ ${ }^{3}$ Maastricht University, Department of Epidemiology, CAPHRI School for Public Health and Primary Care, Maastricht, \\ The Netherlands: ${ }^{4}$ Maastricht University, CARIM School for Cardiovascular Disease, Maastricht, The Netherlands
}

Submitted 26 October 2016: Final revision received 29 March 2017: Accepted 7 April 2017: First published online 31 May 2017

\begin{abstract}
Objective: To find out how the consumption of organic food during pregnancy is associated with consumer characteristics, dietary patterns and macro- and micronutrient intakes.

Design: Cross-sectional description of consumer characteristics, dietary patterns and macro- and micronutrient intakes associated with consumption of organic food during pregnancy.

Setting: Healthy, pregnant women recruited to a prospective cohort study at midwives' practices in the southern part of the Netherlands; to enrich the study with participants adhering to alternative lifestyles, pregnant women were recruited through various specific channels.

Subjects: Participants who filled in questionnaires on food frequency in gestational week 34 ( $n$ 2786). Participant groups were defined based on the share of organic products within various food types.

Results: Consumers of organic food more often adhere to specific lifestyle rules, such as vegetarianism or anthroposophy, than do participants who consume conventional food only (reference group). Consumption of organic food is associated with food patterns comprising more products of vegetable origin (soya/vegetarian products, vegetables, cereal products, bread, fruits, and legumes) and fewer animal products (milk and meat), sugar and potatoes than consumed in conventional diets. These differences translate into distinct intakes of macro- and micronutrients, including higher retinol, carotene, tocopherol and folate intakes, lower intakes of vitamin $\mathrm{D}$ and $\mathrm{B}_{12}$ and specific types of trans-fatty acids in the organic groups. These differences are seen even in groups with low consumption of organic food.

Conclusions: Various consumer characteristics, specific dietary patterns and types of food intake are associated with the consumption of organic food during pregnancy.
\end{abstract}

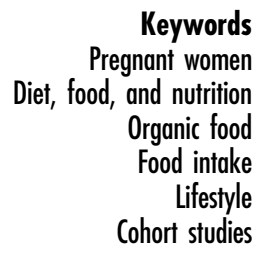

Over the last decades, the consumption of food with an organic certification has increased markedly. To be recognized as organic, food production has to rely on ecological processes, biodiversity and cycles adapted to local conditions, rather than the use of inputs with adverse effects $^{(1)}$ such as synthetic fertilizers and pesticides, preventive veterinary drugs, most preservatives, additives and irradiation. Consumers' motivation to buy organic food is based in part on environmental concerns, but also on the conviction that organic nutrition might promote health. Evidence for the health-promoting effects of organic food for pregnant women and their children is accumulating, and includes associations with a lower risk of preeclampsia $^{(2)}$, of hypospadias in neonates ${ }^{(3)}$ and of atopic eczema and allergic sensitisation in children ${ }^{(4)}$.

When addressing the effect of organic diets on healthrelated parameters in epidemiological and cohort studies, it should be kept in mind that consumers who decide to buy organic food might also exhibit specific sociodemographic characteristics, lifestyles as well as food patterns and intake. The NutriNet-Santé cohort study showed that in France consumption of organic food 
is frequent ${ }^{(5)}$, and regular consumers of organic food are relatively highly educated and physically active in comparison with other food consumer groups ${ }^{(6)}$. A study from the Danish national birth cohort also suggested that organic food consumers have a healthier lifestyle ${ }^{(7)}$. Previous results from the Norwegian large cohort study MoBa showed that consumption of organic food during pregnancy is associated with specific sociodemographic characteristics (low household income, urban dwelling, lowest or highest category of educational attainment), lifestyle characteristics (vegetarian diet, frequent physical exercise) and with favourable health characteristics (normal or low BMI) $^{(8)}$.

A Dutch survey among organic food consumers suggested that the decision to purchase organic food is associated with specific diet characteristics, such as high vegetable consumption, low consumption of ready-made meals and fast food, as well as with the habit of cooking at home $^{(9)}$. A food pattern with more vegetable foods and less sweet and alcoholic beverages, processed meat and milk was also seen in the NutriNet-Santé study ${ }^{(6)}$. The German National Nutrition Survey II revealed that persons who followed an organic diet also consumed more fruits, vegetables and less meat/sausages and soft drinks ${ }^{(10)}$. In Norway, an organic diet during pregnancy was shown to contain more vegetables, fruit and berries, cooking oil, wholegrain products and less meat, white bread, cakes and sweets ${ }^{(11)}$. Furthermore, an organic diet was associated with higher intakes of fibre and some micronutrients, such as folate, $\beta$-carotene and vitamin $\mathrm{C}$, and a low intake of $\mathrm{Na}$.

We hypothesized that the sociodemographic and lifestyle characteristics as well as food pattern and intake of the Dutch KOALA Cohort Study participants who purchased organic food might differ from those of participants who consumed conventional food exclusively. In the present descriptive and explorative study, this hypothesis was tested by defining and comparing participant groups that consumed different proportions of organic products. This work contributes to understanding how the consumption of organic products might influence health.

\section{Participants and methods}

\section{Study design and participants}

The KOALA Birth Cohort Study is a prospective cohort study of 2834 mother-infant pairs in the Netherlands. KOALA is the acronym (in Dutch) for 'Kind, Ouders en Gezondheid: Aandacht voor Leefstijl en Aanleg' (Child, parents and health, addressing lifestyle and constitution).

From October 2000 onwards, healthy, pregnant women ( $n$ 2343) were recruited at midwives' practices in the southern part of the Netherlands participating in an ongoing prospective cohort study on Pregnancy-Related
Pelvic Girdle Pain ${ }^{(12,13)}$. That study did not use lifestylerelated inclusion criteria and the participants showed good comparability with the general population ${ }^{(12)}$ (general population recruitment group). To enrich the KOALA study with participants adhering to an alternative lifestyle, in addition, 491 pregnant women were recruited through various specific channels, such as organic food shops, anthroposophic general practitioners, midwives and underfive clinics, Rudolf Steiner schools, and advertisements in magazines on organic food and other relevant magazines (alternative recruitment group). All women were enrolled at between 14 and 18 weeks of gestation and received detailed questionnaires on sociodemographic and health characteristics at the time of recruitment and at 30 weeks of pregnancy. At 34 weeks of pregnancy, an additional questionnaire was sent to the women dealing with lifestyle and dietary habits, including an FFQ. This questionnaire also enquired about organic food purchase and consumption. Mothers of children who were stillborn or died as neonates ( $n$ 9) or who had Down's syndrome ( $n 7)$ were excluded. In addition, women who had not provided sufficient FFQ data to permit food and nutrient intake analysis ( $n 32$ ) were dropped from the study. The present analysis was therefore based on data from 2786 women.

\section{Measurements}

From the questionnaire administered at week 34, answers to the questions dealing with the following topics were used.

1. Origin (conventional or organic) of seven food groups: (i) meat; (ii) eggs; (iii) vegetables; (iv) fruit; (v) milk and milk products; (vi) bread; and (vii) dried food (dried legumes and cereals). For each of these food groups, we asked participants to report the percentage purchased that was of organic origin: less than $50 \%$, between $50 \%$ and $90 \%$, or more than $90 \%$.

2. Adherence to particular lifestyles rules, including vegetarianism, veganism and anthroposophy.

3. The store(s) where each participant mostly purchased her food, i.e. supermarket, public market, farmers' market, health food shop, organic food shop or specialty shop (bakery, greengrocery, etc.); deliberate purchase of products of organic origin with the EKO label complying with the EU-Eco-regulation 1991 (EEC No. 2092/91) on organic production of agricultural products and their labelling; and deliberate purchase of food products with the Demeter label (biologicdynamic).

4. Purchase of: raw milk; probiotic products (with live bacteria added, such as Yakult, Vifit, Actimel, Biogarde plus, LC1 Nestlé); products with added vitamins or minerals; products sweetened with artificial sweeteners; products sweetened with apple syrup (Diksap), malted barley syrup or honey; products free from additives (colorants, E-numbers, etc.); and products free from GM food components. 


\section{Habitual food and nutrient intakes}

Intakes of food products and nutrients were assessed based on a 198-item FFQ that is an extended version of a previously existing, validated $\mathrm{FFQ}^{(14)}$. For each of the surveyed food products, the frequency of use (nine categories) during the previous month had to be recalled and noted down. In addition, the average amount consumed per day was asked for, in grams (e.g. grams of meat), natural units (e.g. number of apples, slices of bread) or household units (e.g. glasses of milk, spoonfuls of rice). Relative frequency of use of food subtypes was surveyed for some of the foods. The average daily dietary intakes of energy $(\mathrm{kJ})$, macronutrients $(\mathrm{g})$ and micronutrients $(\mathrm{mg}, \mu \mathrm{g})$ were calculated by combining, for each FFQ item, the frequency and amount data with standard portion sizes (g) and with the nutrient composition per $100 \mathrm{~g}$ of food as documented in the 2010 online version of the Dutch Food Composition Table for about 170 nutrients $^{(15)}$. Supplements were not considered in this calculation.

\section{Definition of organic food groups}

Preliminary analysis revealed that the proportions of food items of organic origin in the seven food groups (meat, eggs, vegetables, fruit, milk and milk products, bread, and dried food) were highly correlated. Therefore, use of organic food was defined on the basis of the questions concerning the origin of these seven food groups and on the percentage of organic origin for all seven food groups taken together, unless the respondent reported not consuming one or more of these food groups; in that case, only the food groups consumed were considered for the definition of organic food use. The following groups were defined: (i) ' $<50 \%$ organic', if some food groups were of organic origin, but not all were reported as being more than 50\% organic; (ii) '50-90\% organic', if in all food groups at least $50 \%$ of consumption was of organic origin, but not $>90 \%$ in all cases; and (iii) ' $>90 \%$ organic', if in all the food groups consumed more than $90 \%$ was of organic origin. The remaining group of participants for whom all food groups consumed were products of conventional origin was regarded as the reference group ('conventional'). Relevant characteristics - sociodemographic characteristics, lifestyle, nutritional special products and supplements, food patterns and intakes, energy intake, macronutrient intakes, micronutrient intakes - were compared among the four groups. To improve readability, focus was placed on differences observed between the organic groups taken together and the reference group.

\section{Food intake based on NEVO level-1 classification}

All 215 items on food products were classified according to the Dutch Food Consumption Table (NEVO) aggregate level 1 (hereafter called 'NEVO-1 classification'), which comprises the following twenty-three groups ${ }^{(15)}$ : (i) potatoes; (ii) alcohol and non-alcoholic beverages; (iii) bread; (iv) miscellaneous food products; (v) eggs; (vi) fruits; (vii) pastry and biscuits; (viii) cereals and cereal products; (ix) vegetables; (x) savoury bread spreads; (xi) cheese; (xii) herbs and spices; (xiii) milk and milk products; (xiv) nuts, seeds and snacks; (xv) legumes; (xvi) clinical formulas; (xvii) mixed dishes; (xviii) soups; (xix) soya products and vegetarian products; (xx) sugar, sweets and sweet sauces; (xxi) fats, oils and savoury sauces; (xxii) fish; and (xxiii) meat, processed meat and poultry. Food products within the same food level-1 group were taken together to obtain the participant's intake of each group (in $\mathrm{g} / \mathrm{d}$ ).

\section{Statistical methods}

Principal component analysis (PCA) of all 215 food items (each as g/d) was used to further characterize food patterns. In this approach, correlations between original food intake variables are calculated and each new component (or pattern) becomes a so-called 'factor loading'. We used the varimax rotation with Kaiser normalisation with the following results: the Kaiser-Meyer-Olkin measure verified the sample adequacy for analysis $(\mathrm{KMO}=0.697)$ and Bartlett's test of sphericity $\left(\chi^{2} \quad(22578)=97229 \cdot 589\right.$, $P=<0.001)$ indicated that correlations between items were sufficiently large for PCA. A scree plot was used to determine the number of components (see online supplementary material, Supplemental Fig. 1).

Differences between participant characteristics for the various organic groups in comparison with the conventional group were evaluated as follows. For continuousscale, sociodemographic characteristics, mean values were calculated and compared. For categorical sociodemographic characteristics, percentages were calculated and compared between groups. To test the trend of increasing or decreasing values over the groups, Kendall's $\tau_{\mathrm{c}}$ for categorical variables was applied, with the groups coded 1 to 4 for the conventional (reference) group and the $<50 \%, 50-90 \%$ and $>90 \%$ organic groups, respectively. For food and nutrient intakes (all continuous variables), linear regression was used with dummy variables indicating the comparison of each organic group with the conventional (reference) group, and the trend over the groups was tested using an index variable coded 1 to 4 (as above) in the linear regression.

As potential covariates in the association between organic groups and the conventional (reference) group, we considered the season in which the questionnaire was filled in, maternal age and parity. Confounding was evaluated by adding these variables to the linear regression model and looking at the change of the regression coefficients. The results for the adjusted model are reported if the change was more than $10 \%$ and if the initial or the adjusted $95 \%$ CI did not include the null value.

Data were analysed with the statistical software package IBM $^{\circledR}$ SPSS $^{\circledR}$ Statistics Version 19.0. Two-tailed $P$ values $<0.05$ were considered statistically significant. 


\section{Results}

Sociodemographic, bealth-related and consumption characteristics of study participants

Whereas in the conventional (reference) group the large majority of participants originated from the general population recruitment group (1626/1719, 94.6\%), in the organic groups a considerable percentage of participants originated from the alternative recruitment group (percentages varying between $32.3 \%$ and $54.5 \%$ ). As expected from the recruitment methods, the majority of the participants from the alternative recruitment group used at least some food of organic origin (389/482, $80.7 \%$ ). Nevertheless, almost a third of the participants from the general population recruitment group consumed at least some food of organic origin (678/2304, 29\%).

Compared with the conventional (reference) group, mothers in the organic groups were older at delivery, had more often delivered at least one child previously and more often had a higher level of education (Table 1). The large majority of the women in all four groups were of Dutch ancestry (data not shown).

Women in the various groups were similar in height, but not in weight (Table 1). The average BMI values were slightly but statistically significantly lower in the organic groups than in the conventional (reference) group. Since the various groups were similar with regard to energy intake, the differences in food intake detected reflect true differences in food patterns and not merely different amounts of energy intake. No significant differences were found with regard to alcohol consumption (data not shown).

More women from the organic groups adhered to certain lifestyle rules, most often related to vegetarianism and/or anthroposophy (Table 2). Furthermore, marked differences in food purchasing behaviour between the various organic groups were found. As shown in Table 2, and in comparison with the conventional (reference) group, the organic groups more often purchased products labelled as 'organic' (EKO/SKAL) or biodynamic (Demeter), raw milk, products sweetened with apple syrup, malted barley syrup or honey, and products free from additives and from GM food components. By contrast, fewer products with added vitamins or minerals and products sweetened with artificial sweeteners were consumed than in the conventional (reference) group. Organic food consumers more often purchased their food in farmers' markets, health-food shops (Reformhaus) and organic food shops, and less often from supermarkets and specialty shops.

No significant differences were found in the intakes of food supplements with fatty acids or Fe (Table 2). Multivitamin supplements containing vitamin $\mathrm{D}$ and $\mathrm{B}_{12}$ appeared to be taken in comparable percentages in all groups. However, the organic groups more often used supplements with low-dose vitamin D compared with the conventional (reference) group, which most often used the recommended high-dose supplements $(10 \mu \mathrm{g}$ vitamin $\mathrm{D} / \mathrm{d})$. By contrast, vitamin $B_{12}$ was more often used in high doses by the organic groups.

\section{Food intakes according to the NEVO-1 classification} Compared with the conventional (reference) group, all organic groups showed significantly higher consumption of bread, eggs, fruits, cereal/cereal products, vegetables, cheese, legumes and soya/vegetarian products, but a lower consumption of potatoes, milk/milk products, sugar/sweets/sweet sauces and meat/meat products/ poultry (Table 3). All these differences remained statistically significant when correcting for mother's age, parity and season in which the questionnaire was filled in, even though the differences in the consumption of vegetables,

Table 1 Baseline characteristics of pregnant women with varying purchases of food of organic $v$. conventional origin, KOALA Birth Cohort Study, The Netherlands, 2000-2002 (n 2786)

\begin{tabular}{|c|c|c|c|c|c|c|c|c|c|}
\hline \multirow[b]{2}{*}{ Characteristic } & \multicolumn{2}{|c|}{$\begin{array}{c}\text { Conventional } \\
(n \text { 1719) }\end{array}$} & \multicolumn{2}{|c|}{$\begin{array}{c}<50 \% \text { organic } \\
(n 843)\end{array}$} & \multicolumn{2}{|c|}{$\begin{array}{c}50-90 \% \\
(n 132)\end{array}$} & \multicolumn{2}{|c|}{$\begin{array}{c}>90 \% \text { organic } \\
(n 92)\end{array}$} & \multirow{2}{*}{$\frac{\text { Trend }}{P}$} \\
\hline & Mean or $n$ & SD or $\%$ & Mean or $n$ & SD or $\%$ & Mean or $n$ & SD or $\%$ & Mean or $n$ & SD or $\%$ & \\
\hline Age at delivery (years) & 31.6 & 3.56 & $32 \cdot 4$ & 4.02 & 33.6 & $4 \cdot 15$ & 33.7 & 4.40 & $<0.001$ \\
\hline Number of previous children & & & & & & & & & $<0.001$ \\
\hline 0 & 801 & $46 \cdot 6$ & 353 & $42 \cdot 0$ & 42 & 31.8 & 37 & $40 \cdot 2$ & \\
\hline 1 & 708 & $41 \cdot 2$ & 348 & $41 \cdot 3$ & 61 & $46 \cdot 2$ & 38 & $41 \cdot 3$ & \\
\hline 2 or more & 210 & $12 \cdot 2$ & 142 & $16 \cdot 7$ & 29 & $22 \cdot 0$ & 17 & 18.5 & \\
\hline Highest education & & & & & & & & & $<0.001$ \\
\hline Lower & 1 & 0.1 & 3 & 0.4 & 1 & 0.8 & 0 & 0.0 & \\
\hline Lower-middle (vocational) & 186 & $10 \cdot 8$ & 74 & $8 \cdot 8$ & 13 & 9.8 & 4 & $4 \cdot 3$ & \\
\hline Mid-higher (vocational) & 705 & $41 \cdot 0$ & 282 & 33.5 & 33 & $25 \cdot 0$ & 25 & $27 \cdot 2$ & \\
\hline Higher (high vocational or academic) & 745 & 43.3 & 437 & $51 \cdot 8$ & 79 & 59.8 & 58 & 63.0 & \\
\hline Others & 82 & $4 \cdot 8$ & 47 & $5 \cdot 6$ & 6 & 4.5 & 5 & 5.4 & \\
\hline Height $(\mathrm{cm})$ & 170 & $6 \cdot 2$ & 170 & 6.5 & 170 & $6 \cdot 2$ & 171 & $6 \cdot 3$ & 0.122 \\
\hline Weight before pregnancy (kg) & 69 & $12 \cdot 4$ & 67 & $11 \cdot 2$ & 67 & 12.5 & 68 & $12 \cdot 1$ & 0.004 \\
\hline BMI before pregnancy $\left(\mathrm{kg} / \mathrm{m}^{2}\right)$ & 23.8 & 4.0 & $22 \cdot 9$ & $3 \cdot \overline{6}$ & 22.5 & 4.4 & $22 \cdot 6$ & $3 \cdot 7$ & $<0.001$ \\
\hline Energy intake at gestational week $34(\mathrm{~kJ} / \mathrm{d})$ & 10517 & 2565 & 10730 & 2611 & 10593 & 2435 & 10336 & 2668 & 0.527 \\
\hline
\end{tabular}

Significant $P$ values are indicated in bold font. 
Table 2 Lifestyle, consumer characteristics and special products and supplements taken by pregnant women with varying purchases of food of organic $v$. conventional origin, KOALA Birth Cohort Study, The Netherlands, 2000-2002 (n 2786).

\begin{tabular}{|c|c|c|c|c|c|c|c|c|c|}
\hline \multirow[b]{2}{*}{ Characteristic } & \multicolumn{2}{|c|}{$\begin{array}{l}\text { Conventional } \\
\quad(n 1719)\end{array}$} & \multicolumn{2}{|c|}{$\begin{array}{l}<50 \% \text { organic } \\
\quad(n 843)\end{array}$} & \multicolumn{2}{|c|}{$\begin{array}{l}50-90 \% \text { organic } \\
(n 132)\end{array}$} & \multicolumn{2}{|c|}{$\begin{array}{l}>90 \% \text { organic } \\
\quad(n 92)\end{array}$} & \multirow{2}{*}{$\frac{\text { Trend }}{P}$} \\
\hline & $n$ & $\%$ & $n$ & $\%$ & $n$ & $\%$ & $n$ & $\%$ & \\
\hline Adheres to lifestyle rules* & 19 & $1 \cdot 1$ & 107 & $12 \cdot 9$ & 42 & $32 \cdot 3$ & 32 & $35 \cdot 2$ & $<0.001$ \\
\hline Identifies with anthroposophy & 3 & 0.2 & 34 & $4 \cdot 0$ & 21 & $15 \cdot 9$ & 12 & $13 \cdot 0$ & $<0.001$ \\
\hline Vegetarianism & 14 & 0.9 & 61 & $7 \cdot 3$ & 21 & $15 \cdot 9$ & 21 & $22 \cdot 8$ & $<0.001$ \\
\hline Veganism & 1 & 0.1 & 1 & 0.1 & 0 & 0.0 & 0 & 0.0 & 0.816 \\
\hline \multicolumn{10}{|l|}{ Place where food products are mostly purchased $\dagger$} \\
\hline Supermarket & 1715 & $99 \cdot 8$ & 818 & $97 \cdot 0$ & 110 & 83.3 & 60 & $65 \cdot 2$ & $<0.001$ \\
\hline Public market & 301 & $17 \cdot 5$ & 173 & $20 \cdot 5$ & 12 & $9 \cdot 1$ & 13 & $14 \cdot 1$ & 0.938 \\
\hline Farmers' market & 9 & 0.5 & 20 & 2.4 & 10 & $7 \cdot 6$ & 10 & $10 \cdot 9$ & $<0.001$ \\
\hline Health-food shop (Reformhaus) & 13 & 0.8 & 44 & $5 \cdot 2$ & 8 & $6 \cdot 1$ & 4 & $4 \cdot 3$ & $<0.001$ \\
\hline Organic food shop & 16 & 0.9 & 186 & $22 \cdot 1$ & 64 & 48.5 & 43 & $46 \cdot 7$ & $<0.001$ \\
\hline Specialty shop & 870 & $50 \cdot 6$ & 334 & $39 \cdot 6$ & 42 & $31 \cdot 8$ & 20 & $21 \cdot 7$ & $<0.001$ \\
\hline EKO (yes) $\ddagger$ & 276 & $16 \cdot 1$ & 476 & $56 \cdot 5$ & 96 & $72 \cdot 7$ & 52 & $56 \cdot 5$ & $<0.001$ \\
\hline Demeter (yes) $\ddagger$ & 76 & 4.4 & 317 & $37 \cdot 6$ & 77 & $58 \cdot 3$ & 43 & $46 \cdot 7$ & $<0.001$ \\
\hline Raw milk (yes) $\ddagger$ & 16 & 0.9 & 24 & $2 \cdot 8$ & 5 & $3 \cdot 8$ & 4 & $4 \cdot 3$ & 0.038 \\
\hline Probiotic products (daily) $\ddagger$ & 35 & $2 \cdot 0$ & 24 & $2 \cdot 8$ & 3 & $2 \cdot 3$ & 4 & $4 \cdot 3$ & $0 \cdot 279$ \\
\hline Products with added vitamins or minerals (often) $\ddagger$ & 198 & 11.5 & 78 & $9 \cdot 3$ & 8 & $6 \cdot 1$ & 7 & $7 \cdot 6$ & $<0.001$ \\
\hline Products sweetened with artificial sweeteners (often) $\ddagger$ & 170 & 9.9 & 67 & $7 \cdot 9$ & 12 & $9 \cdot 1$ & 6 & $6 \cdot 5$ & $<0.001$ \\
\hline $\begin{array}{l}\text { Products sweetened with apple syrup (Diksap), malted } \\
\text { barley syrup or honey (often) } \ddagger\end{array}$ & 39 & $2 \cdot 3$ & 87 & $10 \cdot 3$ & 27 & $20 \cdot 5$ & 20 & $21 \cdot 7$ & $<0.001$ \\
\hline $\begin{array}{l}\text { Products free from additives, such as colorants and } \\
\text { E-numbers (often) } \ddagger\end{array}$ & 57 & 3.3 & 140 & $16 \cdot 6$ & 40 & $30 \cdot 3$ & 12 & $13 \cdot 0$ & $<0.001$ \\
\hline $\begin{array}{l}\text { Products free from GM food components (often) } \ddagger \\
\text { Fatty acid supplements } \delta\end{array}$ & 24 & 1.4 & 101 & $12 \cdot 0$ & 25 & 18.9 & 7 & $7 \cdot 6$ & $\begin{array}{r}<0.001 \\
0.287\end{array}$ \\
\hline Fish oil (EPA and DHA) & 4 & & 4 & & 1 & & 1 & & \\
\hline Combined fish and vegetable oil & 2 & & 6 & & 0 & & 0 & & \\
\hline Vegetable oil & 3 & & 1 & & 0 & & 0 & & \\
\hline Vitamin D supplements & & & & & & & & & $<0.001$ \\
\hline None & 632 & $36 \cdot 8$ & 333 & $39 \cdot 5$ & 56 & $42 \cdot 4$ & 36 & $39 \cdot 1$ & \\
\hline Low-dose\| & 162 & 9.4 & 128 & $15 \cdot 2$ & 27 & 20.5 & 20 & $21 \cdot 7$ & \\
\hline High-doseף & 925 & $53 \cdot 8$ & 382 & $45 \cdot 3$ & 49 & $37 \cdot 1$ & 36 & $39 \cdot 1$ & \\
\hline Vitamin $B_{12}$ supplements & & & & & & & & & $<0.001$ \\
\hline None $(0)$ & 91 & $5 \cdot 3$ & 44 & $5 \cdot 2$ & 9 & $6 \cdot 8$ & 6 & $6 \cdot 5$ & \\
\hline Low-dose $(<100 \mu \mathrm{g})^{\star \star}$ & 1572 & 91.4 & 728 & $86 \cdot 4$ & 102 & $77 \cdot 3$ & 72 & $78 \cdot 3$ & \\
\hline High-dose $(\geq 100 \mu \mathrm{g})^{\star \star}$ & 56 & $3 \cdot 3$ & 71 & $8 \cdot 4$ & 21 & $15 \cdot 9$ & 14 & $15 \cdot 2$ & \\
\hline Fe supplements & & & & & & & & & 0.198 \\
\hline None (0) & 477 & $27 \cdot 7$ & 235 & $27 \cdot 9$ & 38 & $28 \cdot 8$ & 23 & $25 \cdot 0$ & \\
\hline$<15 \mathrm{mg} / \mathrm{d} \dagger \dagger$ & 229 & $13 \cdot 3$ & 138 & $16 \cdot 4$ & 20 & $15 \cdot 2$ & 20 & $21 \cdot 7$ & \\
\hline$\geq 15 \mathrm{mg} / \mathrm{d} \neq \ddagger$ & 1013 & 58.9 & 470 & $55 \cdot 8$ & 74 & $56 \cdot 1$ & 49 & $53 \cdot 3$ & \\
\hline
\end{tabular}

Significant $P$ values are indicated in bold font.

*Missing data for adheres to lifestyle rules: $n 8, n 2, n 2$ and $n 1$ for conventional, $<50 \%$ organic, $50-90 \%$ organic and $>90 \%$ organic, respectively. †Participants were allowed to tick two options at most.

fln the FFQ, the corresponding questions allowed respondents to tick different frequencies of consumption. Only one of the possible answers is shown (e.g. yes, often, sometimes) in the table; the $P$ value, however, applies to the whole variable.

§Absolute numbers only.

Multivitamin with $<100 \%$ vitamin D.

\100\% of recommended daily intake of vitamin $\mathrm{D}(100 \%=10 \mu \mathrm{g})$ or higher (in case of combination with multivitamin tablets).

${ }^{\star *}$ Included in high-dose multivitamin preparations.

††The recommended daily amount in the Netherlands in the study period was $14 \mathrm{~g} / \mathrm{d}^{(30)}$, whereas it is now $15 \mathrm{mg} / \mathrm{d}$ for adult women.

$\ddagger \ddagger G$ ravitamon, Matrilon or Fe-only monopreparations, all specially designed for pregnant women. Gravitamon and Matrilon contain 15 mg per daily dosage

$(=114 \%$ of recommended daily intake), whereas Fe monosupplements for pregnant women typically contain $>100 \%$ of the recommended daily intake.

cereal/cereal products, bread, cheese, legumes and eggs in the three organic groups compared with the conventional (reference) group were slightly smaller. Similar smaller differences were observed for milk/milk products and potatoes (Table 3).

\section{Food patterns based on principal component analysis}

Based on PCA, we identified nine food components (see online supplementary material, Supplemental Fig. 1 with the corresponding scree plot) that together explained
$15.2 \%$ of the food items' variance. The components were named 'alternative/vegetarian', 'fast food', 'cooked vegetables', 'raw vegetables salad', 'fish', 'Italian-like cuisine', 'meat', 'traditional stews' and 'sweets', and the corresponding food items are described in Box 1 (in order of decreasing factor loading, if absolute value of the factor loading is greater than 0.30 ).

When we compared the average component scores for these nine components between the various organic groups and the conventional (reference) group, the scores of component 1 ('alternative/vegetarian') and component 4 
Table 3 Food intake $(\mathrm{g} / \mathrm{d})$ according to NEVO level-1 classification in groups of pregnant women with varying purchases of food with organic v. conventional origin, KOALA Birth Cohort Study, The Netherlands, 2000-2002 (n 2786)

\begin{tabular}{|c|c|c|c|c|c|c|c|c|c|}
\hline \multirow[b]{2}{*}{ NEVO-1 food group*, $\dagger(\mathrm{g} / \mathrm{d})$} & \multicolumn{2}{|c|}{$\begin{array}{c}\text { Conventional (reference) } \\
\text { group } \\
(n 1719)\end{array}$} & \multicolumn{2}{|c|}{$\begin{array}{l}<50 \% \text { organic } \\
(n 843)\end{array}$} & \multicolumn{2}{|c|}{$\begin{array}{c}50-90 \% \text { organic } \\
(n 132)\end{array}$} & \multicolumn{2}{|c|}{$\begin{array}{l}>90 \% \text { organic } \\
(n 92)\end{array}$} & \multirow{2}{*}{$\frac{\text { Trend } \neq}{P}$} \\
\hline & Mean & SD & $\beta$ & $95 \% \mathrm{Cl}$ & $\beta$ & $95 \% \mathrm{Cl}$ & $\beta$ & $95 \% \mathrm{Cl}$ & \\
\hline \multicolumn{10}{|l|}{ 1. Potatoes } \\
\hline \multicolumn{10}{|c|}{ 2. Alcohol and non-alcoholic beverages } \\
\hline $\begin{array}{l}\text { Crude } \\
\text { Adjusted } \\
\text { 3. Bread }\end{array}$ & 1240 & 524 & $\begin{array}{l}47 \cdot 6 \\
52 \cdot 2\end{array}$ & $\begin{array}{r}5 \cdot 7,89 \cdot 5 \\
10 \cdot 1,94 \cdot 3\end{array}$ & $\begin{array}{l}26 \cdot 8 \\
40 \cdot 4\end{array}$ & $\begin{array}{l}-63 \cdot 3,116 \cdot 9 \\
-125 \cdot 9,87 \cdot 9\end{array}$ & $\begin{array}{l}-19 \cdot 3 \\
-39 \cdot 9\end{array}$ & $\begin{array}{r}-126 \cdot 0,87 \cdot 5 \\
-89 \cdot 8,10 \cdot 0\end{array}$ & $\begin{array}{l}0.287 \\
0.243\end{array}$ \\
\hline $\begin{array}{l}\text { Crude } \\
\text { Adjusted } \\
\text { 4. Miscellaneous food prod }\end{array}$ & 165 & 59 & $\begin{array}{l}6.7 \\
6.0\end{array}$ & $\begin{array}{l}1.7,11.8 \\
0.9,11 \cdot 1\end{array}$ & $\begin{array}{l}15 \cdot 2 \\
13 \cdot 5\end{array}$ & $\begin{array}{l}4 \cdot 3,26 \cdot 1 \\
2 \cdot 6,24 \cdot 5\end{array}$ & $\begin{array}{l}5 \cdot 8 \\
4 \cdot 9\end{array}$ & $\begin{array}{l}-7 \cdot 0,18 \cdot 7 \\
-8 \cdot 0,17 \cdot 9\end{array}$ & $\begin{array}{l}0.003 \\
0.008\end{array}$ \\
\hline $\begin{array}{l}\text { Crude } \\
\text { 5. Eggs }\end{array}$ & 0.3 & $4 \cdot 6$ & 1.7 & $0.9,2.4$ & $6 \cdot 1$ & $4 \cdot 4,7 \cdot 7$ & 2.7 & $0.7,4.7$ & $<0.001$ \\
\hline $\begin{array}{l}\text { Crude } \\
\text { Adjusted } \\
\text { 6. Fruits }\end{array}$ & 8.5 & $7 \cdot 8$ & $1 \cdot 1$ & $\begin{array}{l}0.5,1.8 \\
-\end{array}$ & $\begin{array}{l}2 \cdot 2 \\
2 \cdot 1\end{array}$ & $\begin{array}{l}0.8,3.6 \\
0.7,3.6\end{array}$ & 0.3 & $\begin{array}{l}-1 \cdot 4,2 \cdot 0 \\
-\end{array}$ & $\begin{array}{l}0.002 \\
0.005\end{array}$ \\
\hline $\begin{array}{l}\text { Crude } \\
\text { Adjusted } \\
\text { 7. Pastry and biscuits }\end{array}$ & 143 & 68 & $17 \cdot 5$ & $\begin{array}{l}11 \cdot 9,23 \cdot 1 \\
-\end{array}$ & $\begin{array}{l}14.8 \\
16 \cdot 6\end{array}$ & $\begin{array}{l}2 \cdot 8,26 \cdot 8 \\
4 \cdot 5,28 \cdot 8\end{array}$ & $7 \cdot 0$ & $\begin{array}{l}-7 \cdot 3,21 \cdot 3 \\
-\end{array}$ & $\begin{array}{l}<0.001 \\
<0.001\end{array}$ \\
\hline $\begin{array}{l}\text { Crude } \\
\text { Adjusted } \\
\text { 8. Cereals and cereal produ }\end{array}$ & 41 & 28 & $\begin{array}{l}0.6 \\
0.1\end{array}$ & $\begin{array}{l}-1 \cdot 7,3 \cdot 0 \\
-2 \cdot 2,2 \cdot 4\end{array}$ & $\begin{array}{l}-1 \cdot 1 \\
-2 \cdot 5\end{array}$ & $\begin{array}{l}-6 \cdot 1,3 \cdot 9 \\
-7 \cdot 5,2 \cdot 5\end{array}$ & $\begin{array}{l}-2 \cdot 6 \\
-3 \cdot 5\end{array}$ & $\begin{array}{l}-8 \cdot 6,3 \cdot 3 \\
-9 \cdot 4,2 \cdot 4\end{array}$ & $\begin{array}{l}0.611 \\
0.274\end{array}$ \\
\hline $\begin{array}{l}\text { Crude } \\
\text { Adjusted } \\
\text { 9. Vegetables }\end{array}$ & 57 & 39 & 11.7 & ${ }_{-}^{8 \cdot 1,15 \cdot 2}$ & 19.9 & $\begin{array}{l}12 \cdot 3,27 \cdot 5 \\
-\end{array}$ & $\begin{array}{l}23.0 \\
20.2\end{array}$ & $\begin{array}{l}14 \cdot 0,32 \cdot 1 \\
11 \cdot 2,29 \cdot 3\end{array}$ & $\begin{array}{l}<0.001 \\
<0.001\end{array}$ \\
\hline $\begin{array}{l}\text { Crude } \\
\text { Adjusted }\end{array}$ & 158 & 109 & $\begin{array}{l}17 \cdot 3 \\
15 \cdot 6\end{array}$ & $\begin{array}{l}9 \cdot 3,25 \cdot 3 \\
7 \cdot 6,23 \cdot 6\end{array}$ & $\begin{array}{l}24 \cdot 3 \\
19 \cdot 3\end{array}$ & $\begin{array}{l}7 \cdot 1,41 \cdot 4 \\
2 \cdot 2,36 \cdot 5\end{array}$ & $\begin{array}{l}24 \cdot 0 \\
17 \cdot 7\end{array}$ & $\begin{array}{r}3 \cdot 7,44 \cdot 3 \\
-2 \cdot 6,38 \cdot 0\end{array}$ & $\begin{array}{l}<0.001 \\
<0.001\end{array}$ \\
\hline $\begin{array}{l}\text { 10. Savoury bread spreads } \\
\text { Crude } \\
\text { 11. Cheese }\end{array}$ & $3 \cdot 1$ & $5 \cdot 8$ & 2.0 & $1.4,2.6$ & 2.9 & $1 \cdot 6,4 \cdot 2$ & 1.8 & $0.3,3.3$ & $<0.001$ \\
\hline $\begin{array}{l}\text { Crude } \\
\text { Adjusted } \\
\text { 13. Milk and milk products }\end{array}$ & 29 & 25 & $\begin{array}{l}6 \cdot 2 \\
5 \cdot 4\end{array}$ & $\begin{array}{l}4 \cdot 1,8 \cdot 4 \\
3 \cdot 2,7 \cdot 5\end{array}$ & $\begin{array}{l}9.6 \\
7.5\end{array}$ & $\begin{array}{l}5 \cdot 0,14 \cdot 3 \\
2 \cdot 8,12 \cdot 1\end{array}$ & $\begin{array}{l}7 \cdot 7 \\
5 \cdot 3\end{array}$ & $\begin{array}{r}2 \cdot 2,13 \cdot 2 \\
-0 \cdot 2,10 \cdot 8\end{array}$ & $\begin{array}{l}<0.001 \\
<0.001\end{array}$ \\
\hline $\begin{array}{l}\text { Crude } \\
\text { Adjusted } \\
\text { 14. Nuts, seeds and snacks }\end{array}$ & 423 & 246 & $-13 \cdot 3$ & $\begin{array}{l}-34 \cdot 0,7 \cdot 4 \\
-\end{array}$ & $\begin{array}{l}-69.0 \\
-62.4\end{array}$ & $\begin{array}{l}-113 \cdot 5,-24 \cdot 6 \\
-107 \cdot 1,-17 \cdot 7\end{array}$ & $-76 \cdot 2$ & $\begin{array}{l}-128 \cdot 9,-23.5 \\
-\end{array}$ & $\begin{array}{l}<0.001 \\
<0.001\end{array}$ \\
\hline $\begin{array}{l}\text { Crude } \\
\text { Adjusted }\end{array}$ & 23 & 21 & $\begin{array}{r}-0.1 \\
0.1\end{array}$ & $\begin{array}{l}-1 \cdot 8,1 \cdot 6 \\
-1 \cdot 6,1.9\end{array}$ & $\begin{array}{r}-0.1 \\
0.4\end{array}$ & $\begin{array}{l}-3 \cdot 8,3 \cdot 6 \\
-3 \cdot 3,4 \cdot 2\end{array}$ & $\begin{array}{l}-3 \cdot 6 \\
-3.1\end{array}$ & $\begin{array}{l}-8 \cdot 0,0 \cdot 8 \\
-7 \cdot 6,1 \cdot 3\end{array}$ & $\begin{array}{l}0.277 \\
0.446\end{array}$ \\
\hline \multicolumn{10}{|l|}{ 15. Legumes } \\
\hline \multicolumn{10}{|l|}{ 17. Mixed dishes } \\
\hline $\begin{array}{l}\text { Crude } \\
\text { 18. Soups }\end{array}$ & 40 & 28 & $-3 \cdot 0$ & $-5 \cdot 3,-0 \cdot 7$ & $-2 \cdot 1$ & $-7 \cdot 0,2 \cdot 8$ & -7.5 & $-13 \cdot 3,-1 \cdot 6$ & 0.002 \\
\hline $\begin{array}{l}\text { Crude } \\
\text { Adjusted }\end{array}$ & 68 & 75 & $\begin{array}{l}8 \cdot 8 \\
8.4\end{array}$ & $\begin{array}{l}2 \cdot 6,15 \cdot 0 \\
2 \cdot 2,14 \cdot 6\end{array}$ & $\begin{array}{l}-1 \cdot 3 \\
-2 \cdot 9\end{array}$ & $\begin{array}{l}-14 \cdot 6,12 \cdot 0 \\
-16 \cdot 2,10 \cdot 5\end{array}$ & $\begin{array}{l}-0.6 \\
-1.5\end{array}$ & $\begin{array}{l}-16 \cdot 4,15 \cdot 1 \\
-17 \cdot 3,14 \cdot 3\end{array}$ & $\begin{array}{l}0.255 \\
0.312\end{array}$ \\
\hline \multicolumn{10}{|c|}{ 19. Soya products and vegetarian products } \\
\hline \multicolumn{8}{|c|}{ 20. Sugar, sweets and sweet sauces } & $8 \cdot 3,20 \cdot 8$ & $<0.001$ \\
\hline $\begin{array}{l}\text { Crude } \\
\text { 21. Fats, oils and savoury sa }\end{array}$ & 96 & \multicolumn{7}{|c|}{ 21. Fats, oils and savoury sauces } & $<0.001$ \\
\hline $\begin{array}{l}\text { Crude } \\
\text { 22. Fish }\end{array}$ & 61 & 27 & $-2 \cdot 1$ & $-4 \cdot 4,0.2$ & 2.9 & $-2 \cdot 1,7 \cdot 8$ & 1.5 & $-4 \cdot 4,7 \cdot 3$ & 0.975 \\
\hline $\begin{array}{l}\text { Crude } \\
\text { Adjusted }\end{array}$ & 40 & 36 & $\begin{array}{l}5 \cdot 1 \\
4 \cdot 4\end{array}$ & $\begin{array}{l}1.9,8.2 \\
1.3,7.5\end{array}$ & $\begin{array}{l}-0.3 \\
-1.7\end{array}$ & $\begin{array}{r}-7 \cdot 01,6 \cdot 4 \\
-8 \cdot 4,5 \cdot 1\end{array}$ & $\begin{array}{l}-7 \cdot 3 \\
-9 \cdot 4\end{array}$ & $\begin{array}{r}-15 \cdot 3,0.7 \\
17 \cdot 4,-1.5\end{array}$ & $\begin{array}{l}0.789 \\
0.674\end{array}$ \\
\hline \multicolumn{10}{|c|}{ 23. Meat, processed meat and /poultry } \\
\hline Crude & 107 & 45 & -23.1 & $-27 \cdot 1,-19 \cdot 1$ & $-45 \cdot 0$ & $-53.6,-36.5$ & $-42 \cdot 0$ & $-52 \cdot 1,-31 \cdot 8$ & $<0.001$ \\
\hline
\end{tabular}

Significant results and $P$ values are indicated in bold font.

${ }^{*}$ The NEVO highest level of classification was followed as originally described ${ }^{(15)}$. According to this classification, group 4 (miscellaneous food products) includes tartex and group 8 (cereals and cereal products) comprises seitan. These two products were not originally developed by/for vegetarian cuisine. Therefore, we decided to use the original classification, even though they are often used in the vegetarian diet.

†Data were obtained within two different linear regression models, both based on the consumption of organic food (categorical, index 1-4, reference group is $0 \%$ organic): (i) 'crude model', without additional covariates; and (ii) 'adjusted model' (values shown only when differed from the crude model), adjusted for age, parity and season. Values of $\beta$ (regression coefficient) and $95 \% \mathrm{Cl}$ indicate the difference of the mean values $(\mathrm{g} / \mathrm{d})$ between the organic group and the conventional (reference group) for all group participants.

$\ddagger$ Test-for-trend of increasing or decreasing $\beta$ over the categories of consumption of organic food (coded as $1-4$ for $0 \%$ through $>90 \%$ organic). 
Box 1 Main food pattern components as identified by principal component analysis

1. 'Alternative/vegetarian': positive loadings for tofu/tempeh/soya chunks, vegetarian burger/schnitzel, pumpkin, seitan, butter as cooking fat, vegetarian sandwich spread, sunflower/pumpkin seeds, nuts/raisins mixed, cereals (buckwheat, bulgur, couscous and grits), quorn (small pieces), fennel, butter as spread for bread, crackers, etc., dried/candied fruits, quorn (burger), cheese (as part of a hot meal), beans (kidney beans, white beans, marrowfat beans, lentils); negative loadings for chicken and pork (tenderloin, fricandeau, schnitzel).

2. 'Fast food': positive loadings for chips (as part of a hot meal), mayonnaise, French fries, minced-meat burger, meat croquettes, fried potatoes, solid deep-frying fat as cooking fat, bread rolls/buns (as a cold meal), soft drinks/ syrup, concentrated fruit drink; negative loading for brown bread.

3. 'Cooked vegetables': positive loadings for French beans, cauliflower, broccoli, spinach, carrots, potatoes (boiled), chicory, leek, endive and red cabbage.

4. 'Raw vegetables salad': positive loadings for dressing for raw vegetable salad, raw cabbage, raw lettuce, raw cucumber, raw carrots, raw lamb's tongue lettuce/purslane/water cress, other raw vegetables in salads, raw cabbage and raw sprouts.

5. 'Fish': positive loadings for fish as hot meal, fried/deep-fried fish, salmon steak/fillet, canned fish, white fish fillet, mackerel/eel; and olives as a snack.

6. 'Italian-like cuisine': positive loadings for pasta, oil as cooking fat, tomato sauce/tomato ketchup, minced beef, sweet paprika (as part of a hot meal), onions, ready-made oriental sauce, various hot sauces, various cooked vegetables.

7. 'Meat': positive loadings for salami sausage/saveloy/luncheon meat, boiled ham/fricandeau/cured pork side, luncheon meat sausage/cooked sausage/sausage with smoked bacon-bits, luncheon meat sausage/cooked sausage/ sausage with smoked bacon-bits (cold meal), loaf/fried minced meat, smoke-dried/roast beef/corned beef, rashers streaky bacon/bacon, 'braadworst'/slavink' (Dutch meat specialties) and cutlet/pork steak/pork collops.

8. 'Traditional stews': positive loadings for curly kale, sauerkraut, cabbage, smoked sausage, Brussels sprouts; negative loadings for strawberries.

9. 'Sweets': positive loadings for biscuits/cookies, cake, sweets and chips.

('raw vegetables') were significantly higher in all organic groups relative to the conventional (reference) group, whereas the scores for components 2 ('fast food') and 7 ('meat') were significantly lower in the organic groups (see online supplementary material, Supplemental Table 1). These differences remained statistically significant when mother's age, parity and season were adjusted for.

\section{Intakes of nutrients}

As shown in Table 4, the food pattern in the organic groups translated into significantly higher dietary fibre consumption relative to the conventional (reference) group. The organic groups consumed less protein (total), even though the markedly lower intake of animal protein was to a considerable degree compensated for by higher plant protein intake. No differences in intakes of total carbohydrates and total fat were found.

There were numerous differences in micronutrient intakes between the different groups. A few are shown in Table 4; the specialized reader is referred to the online supplementary material, Supplemental Table 2, for additional values. Relative to the conventional (reference) group, the organic groups showed higher intakes of carotenes ( $\alpha$ and $\beta$ ), tocopherols ( $\alpha, \beta, \gamma$ and $\delta$ ), retinol and folate, but lower intakes of vitamins $B_{12}$ and $D$. The organic groups also had higher intakes of $\mathrm{Mg}$, total Fe and non-haem Fe, but a lower intake of haem Fe. Concerning mono- and disaccharides, intake was higher for fructose and glucose, and lower for sucrose and lactose, in the organic groups compared with the conventional (reference) group.

The total amount of fatty acids consumed was similar in all four groups, and no significant differences were detected for combined MUFA or PUFA (total, $n-3$ and $n-6$; data not shown). However, the amounts of some fatty acids differed between the groups (Table 4, Supplemental Table 2). Intake level of pentadecanoic acid (15:0, abundant in milk products) was higher in the organic groups, whereas intake levels of oleic acid $(18: 1 n-9, c i s)$, dihomo- $\gamma$-linolenic acid $(20: 3 n-6)$ and arachidonic acid $(20: 4 n-6)$ were lower. Total intake of trans-fatty acids was slightly higher in the organic groups. But within this class of fatty acids, intake of elaidic acid (18:1n-9,trans), as the indicator for industrially dehydrogenated (hardened) vegetable oil, was lower in the organic groups, whereas the intake of vaccenic acid (18:1n-7,trans) and conjugated linoleic acids (18: 2 conjugated isomers), as indicators for trans-fatty acids from ruminant origin, were higher (Table 4).

\section{Discussion}

In the present work, we investigated the associations between the use of organic food and various sociodemographic, lifestyle and consumption characteristics, 
Table 4 Intakes of macronutrients and selected micronutrients by pregnant women with varying purchases of food with organic $v$. conventional origin, KOALA Birth Cohort Study, The Netherlands, 2000-2002 ( $n$ 2786)

\begin{tabular}{|c|c|c|c|c|c|c|c|c|c|}
\hline \multirow[b]{2}{*}{ Nutrient* } & \multicolumn{2}{|c|}{$\begin{array}{c}\text { Conventional (reference) } \\
\text { group } \\
(n \text { 1719) } \\
\end{array}$} & \multicolumn{2}{|c|}{$\begin{array}{c}<50 \% \text { organic } \\
(n 843)\end{array}$} & \multicolumn{2}{|c|}{$\begin{array}{c}50-90 \% \text { organic } \\
(n \text { 132) }\end{array}$} & \multicolumn{2}{|c|}{$\begin{array}{l}>90 \% \text { organic } \\
(n 92)\end{array}$} & \multirow{2}{*}{$\frac{\text { Trend } \dagger}{P}$} \\
\hline & Mean & SD & $\beta$ & $95 \% \mathrm{Cl}$ & $\beta$ & $95 \% \mathrm{Cl}$ & $\beta$ & $95 \% \mathrm{Cl}$ & \\
\hline \multicolumn{10}{|l|}{ Water $(\mathrm{g} / \mathrm{d})$} \\
\hline $\begin{array}{l}\text { Crude } \\
\text { Adjusted }\end{array}$ & 2306 & 754 & 136 & $\begin{array}{l}72.8,198.9 \\
-\quad\end{array}$ & $\begin{array}{l}23 \\
16\end{array}$ & $\begin{array}{l}-112 \cdot 9,156 \cdot 0 \\
-119 \cdot 8,151 \cdot 6\end{array}$ & $\begin{array}{r}9 \\
-20\end{array}$ & $\begin{array}{l}-151 \cdot 0,169.9 \\
-179.9,140.8\end{array}$ & $\begin{array}{l}0.031 \\
0.069\end{array}$ \\
\hline \multicolumn{10}{|c|}{ Total protein $(\mathrm{g} / \mathrm{d})$} \\
\hline $\begin{array}{c}\text { Crude } \\
\text { Plant protei }\end{array}$ & \multicolumn{8}{|c|}{ Plant protein (g/d) } & 0.018 \\
\hline $\begin{array}{l}\text { Crude } \\
\text { Adjusted }\end{array}$ & 36 & 10 & 3.8 & ${ }_{-}^{3.0,4.7}$ & $7 \cdot 8$ & $\begin{array}{r}5.9,9.6 \\
-\quad 5\end{array}$ & $\begin{array}{l}5.2 \\
4.6\end{array}$ & $\begin{array}{l}3.0,7 \cdot 3 \\
2 \cdot 4,6 \cdot 8\end{array}$ & $\begin{array}{l}<0.001 \\
<0.001\end{array}$ \\
\hline \multicolumn{10}{|c|}{ Animal protein $(\mathrm{g} / \mathrm{d})$} \\
\hline $\begin{array}{l}\text { Crude } \\
\text { Carbohydra }\end{array}$ & \multicolumn{8}{|c|}{ Carbohydrates (g/d) } & $<0.001$ \\
\hline $\begin{array}{l}\text { Crude } \\
\text { Adjusted }\end{array}$ & 295 & 83 & $\begin{array}{l}4 \cdot 3 \\
5 \cdot 3\end{array}$ & $\begin{array}{l}-2 \cdot 4,11 \cdot 0 \\
-1 \cdot 3,12 \cdot 1\end{array}$ & $\begin{array}{r}-0.2 \\
2.7\end{array}$ & $\begin{array}{l}-14 \cdot 5,14 \cdot 2 \\
-11 \cdot 8,17 \cdot 1\end{array}$ & $\begin{array}{r}-2.4 \\
0.2\end{array}$ & $\begin{array}{l}-19 \cdot 4,14 \cdot 6 \\
-16 \cdot 9,17 \cdot 3\end{array}$ & $\begin{array}{l}0.692 \\
0.413\end{array}$ \\
\hline \multicolumn{10}{|c|}{ Total fat $(\mathrm{g} / \mathrm{d})$} \\
\hline $\begin{array}{l}\text { Crude } \\
\text { Adjusted }\end{array}$ & 98 & 27 & 1.4 & $\begin{array}{r}0.9,3.7 \\
-\quad\end{array}$ & $\begin{array}{l}1.9 \\
1.7\end{array}$ & $\begin{array}{l}-3 \cdot 0,6 \cdot 8 \\
-3 \cdot 2,6 \cdot 6\end{array}$ & $\begin{array}{l}-3.4 \\
-3.9\end{array}$ & $\begin{array}{l}-9 \cdot 4,2 \cdot 1 \\
-9 \cdot 7,1.9\end{array}$ & $\begin{array}{l}0.899 \\
0.926\end{array}$ \\
\hline \multicolumn{10}{|c|}{ Dietary fibre $(g / d)$} \\
\hline $\begin{array}{l}\text { Crude } \\
\text { Adjusted }\end{array}$ & vity equival & 6 & $2 \cdot 2$ & $\begin{array}{r}1 \cdot 6,2 \cdot 7 \\
-\quad\end{array}$ & 4.0 & $\begin{array}{r}2 \cdot 8,5 \cdot 1 \\
-\quad\end{array}$ & $\begin{array}{l}2 \cdot 6 \\
2 \cdot 3\end{array}$ & $\begin{array}{l}1.2,3.4 \\
0.9,3.6\end{array}$ & $\begin{array}{l}<0.001 \\
<0.001\end{array}$ \\
\hline \multicolumn{10}{|c|}{ Retinol (activity equivalents) } \\
\hline $\begin{array}{c}\text { Crude } \\
\beta \text {-Carotene }\end{array}$ & \multicolumn{9}{|c|}{$\beta$-Carotene $(\mu \mathrm{g})$} \\
\hline $\begin{array}{l}\text { Crude } \\
\text { Adjusted }\end{array}$ & 2628 & 1348 & $\begin{array}{l}333.7 \\
302.4\end{array}$ & $\begin{array}{l}220 \cdot 0,447.5 \\
188.6,416.2\end{array}$ & $\begin{array}{l}616.0 \\
534.2\end{array}$ & $\begin{array}{l}371 \cdot 6,860.4 \\
289.4,779.0\end{array}$ & $\begin{array}{l}670.8 \\
596.0\end{array}$ & $\begin{array}{l}381 \cdot 3,960 \cdot 4 \\
306 \cdot 7,885 \cdot 3\end{array}$ & $\begin{array}{l}<0.001 \\
<0.001\end{array}$ \\
\hline \multicolumn{10}{|c|}{ Vitamin D (total, $\mu \mathrm{g})$} \\
\hline $\begin{array}{l}\text { Crude } \\
\text { a-Tocopher }\end{array}$ & (mg) & $1 \cdot 6$ & -0.17 & $-0.3,-0.0$ & -0.65 & $-1 \cdot 0,-0 \cdot 4$ & -0.94 & $-1 \cdot 3,-0.6$ & $<0.001$ \\
\hline \multicolumn{10}{|c|}{ Folate (equivalents) } \\
\hline $\begin{array}{l}\text { Crude } \\
\text { Adjusted }\end{array}$ & 265 & 71 & $22 \cdot 9$ & ${ }^{16 \cdot 9,28.8}$ & 34.6 & $-^{21 \cdot 7,47.5}$ & $\begin{array}{l}25 \cdot 1 \\
21 \cdot 2\end{array}$ & $\begin{array}{l}9.8,40.3 \\
6.0,36.5\end{array}$ & $\begin{array}{l}<0.001 \\
<0.001\end{array}$ \\
\hline \multicolumn{10}{|c|}{ Oleic acid (18: $1 n-9$, cis, mg) } \\
\hline \multicolumn{9}{|c|}{ Elaidic acid (18:1n-9,trans, mg) } & $<0.001$ \\
\hline $\begin{array}{l}\text { Crude } \\
\text { Vaccenic ac }\end{array}$ & $\begin{array}{c}789 \\
\text { id }(18: 1 n-7\end{array}$ & $\begin{array}{r}275 \\
n s, \mathrm{mg})\end{array}$ & -51.9 & $-74 \cdot 7,-29 \cdot 1$ & $-92 \cdot 1$ & $-141 \cdot 0,-43 \cdot 1$ & $-117 \cdot 3$ & $-175 \cdot 3,-59 \cdot 3$ & $<0.001$ \\
\hline $\begin{array}{l}\text { Crude } \\
\text { Adjusted }\end{array}$ & 303 & 182 & $\begin{array}{l}81.4 \\
72.5\end{array}$ & $\begin{array}{r}63.5,99 \cdot 3 \\
54.9,90 \cdot 3\end{array}$ & $\begin{array}{l}186 \cdot 1 \\
163 \cdot 2\end{array}$ & $\begin{array}{l}147 \cdot 6,224 \cdot 6 \\
125 \cdot 1,201 \cdot 2\end{array}$ & $\begin{array}{l}143.6 \\
120.8\end{array}$ & $\begin{array}{l}98 \cdot 0,189 \cdot 2 \\
75 \cdot 8,165 \cdot 8\end{array}$ & $\begin{array}{l}<0.001 \\
<0.001\end{array}$ \\
\hline \multicolumn{10}{|c|}{ Conjugated linoleic acids (18: 2 conjugated isomers, total, $\mathrm{mg}) \ddagger$} \\
\hline Crude & 53 & 34 & 18.5 & $14 \cdot 8,22 \cdot 1$ & 43.0 & $35 \cdot 1,51 \cdot 0$ & 35.4 & $26 \cdot 0,44 \cdot 8$ & $<0.001$ \\
\hline
\end{tabular}

Significant results and $P$ values are indicated in bold font.

*Data were obtained within two different linear regression models, both based on the consumption of organic food (categorical, index 1-4, reference group is $0 \%$ organic): (i) 'crude model', without additional covariates; and (ii) 'adjusted model' (values shown only when differed from the crude model), adjusted for age, parity and season. Values of $\beta$ (regression coefficient) and $95 \% \mathrm{Cl}$ indicate the difference of the mean values between the organic group and the conventional (reference group) for all group participants, expressed $\mathrm{g} / \mathrm{d}$ for macronutrients, or as $\mathrm{mg} / \mathrm{d}, \mu \mathrm{g} / \mathrm{d}$ for micronutrients. Results on 25 -hydroxyvitamin D were comparable to those on vitamin D (total); results on $\beta$-carotene were comparable to those on a-carotene; results on a-tocopherol were comparable to those on $\beta$-tocopherol, $\gamma$-tocopherol and $\delta$-tocopherol. Nutrient values were calculated with basis on the data from the FFQ only; supplements were not considered. †Test-for-trend of increasing or decreasing $\beta$ over the categories of consumption of organic food (coded as $1-4$ for $0 \%$ through $>90 \%$ organic).

¥Mainly rumenic acid (18:2,n-7,trans, $n-9$, cis).

as well as dietary patterns in a large Dutch population of pregnant women. We performed this analysis within the KOALA Birth Cohort Study, a cohort study on pregnant women and their offspring. In the present study, participants with an alternative lifestyle were actively recruited with the purpose of increasing contrast in related exposure variables, including nutrition. This strategy enabled a deeper insight into the characteristics of this specific part of the population and allowed us to consider the proportion of organic food in our analysis. Nevertheless, the large majority of the participants were recruited independently of their lifestyle (general population recruitment group, $83 \%$ of all participants).

\section{Characteristics associated with the consumption of organic food}

Our study reveals various relevant lifestyle-related differences between organic food consumers and non-consumers. Similarly to what has been previously reported in France ${ }^{(6)}$, the organic food consumers in our study had more often 
completed higher education. Furthermore, they more frequently followed an alternative lifestyle (influenced by lifestyle rules such as those related to anthroposophy and vegetarianism) than did non-consumers of organic food. Although no differences in daily energy intake were detected, organic food consumers had a statistically significantly lower BMI than the rest of the participants. The difference can be seen as small but, in combination with the fact that energy intake was similar, it might indicate a more active lifestyle, as has also been reported in the French NutriNet-Santé study ${ }^{(6)}$ and in the Norwegian large cohort study $\mathrm{MoBa}^{(8)}$.

In all groups, most food was bought in supermarkets but, as was to be expected from the recruitment methods, organic food consumers bought more food in organic food shops and farmers' markets than did non-consumers. Products with industrially produced components, such as added vitamins or artificial sweeteners, were consumed less often in the organic consumer groups who, in contrast, more often bought products with natural sweeteners, free from additives and free from GM food components than did conventional consumers. All groups consumed vitamin $\mathrm{D}$ and vitamin $\mathrm{B}_{12}$ supplements frequently. In the organic groups, low-dose vitamin $\mathrm{D}$ was preferred - perhaps organic consumers think they have a lifestyle with more sunshine exposure - but high-dose vitamin $\mathrm{B}_{12}$ supplements were more often consumed. The latter might be associated with the higher percentage of vegetarian participants in the organic groups.

\section{Food patterns and intakes}

Data from FFQ were analysed with three different approaches to food patterns and intakes: (i) by characterizing associations with food-related characteristics such as vegetarianism (see above); (ii) by grouping food components into broad food groups according to the NEVO-1 classification; and (iii) by performing a PCA on all food items. The results obtained following the various approaches are in line with each other. They show that the consumption of organic food is associated with specific consumer behaviours, food patterns and intakes. The latter comprise more products of vegetable origin - soya/ vegetarian products, vegetables, cereal and cereal products, bread, fruits, legumes - and fewer animal products - milk and milk products, meat and meat products - than conventional diets do. In addition, the consumption of sugar, sweets and sweet sauces as well as of potatoes was lower among the participants with an at least partially organic diet. Consumption of more organic food was associated with fewer elements typical of fast food (the second component in the PCA) and of traditional food, with 'stamppot' being a well-known example of traditional Dutch food (included in component 8).

Despite the markedly different food patterns and intakes in the various groups, the corresponding energy intakes were similar. This observation differs from the results reported from the MoBa study, which revealed higher energy intakes in organic groups in Norway ${ }^{(11)}$. The calculated intakes of the various macronutrients revealed substitution for animal protein with plant protein, which is in agreement with the higher amounts of fibre in the organic groups. The micronutrients analysis revealed several examples of higher intakes of possibly healthpromoting substances in the organic groups relative to the conventional (reference) group. This is the case for $\mathrm{Ca}$ and $\mathrm{Mg}$, as well as for retinol, $\alpha$ - and $\beta$-carotenes, $\alpha$-, $\beta$-, $\gamma$ - and $\delta$-tocopherols, and folate.

In some forms of vegetarianism, special care should be taken that sufficient amounts of vitamin $\mathrm{B}_{12}$, vitamin $\mathrm{D}, n-3$ fatty acids, $\mathrm{Ca}, \mathrm{Fe}$ and $\mathrm{Zn}$ are present in the diet ${ }^{(16)}$. Our results revealed that in the organic groups, in which the consumption of animal products was limited, only the dietary intakes of vitamin $\mathrm{B}_{12}$ and vitamin $\mathrm{D}$ were lower than in the conventional (reference) group, whereas the intake of Ca was even higher. Although the differences were modest, it is conceivable that in some individuals supplements would have been advisable. Concerning $\mathrm{Fe}$, it is interesting to note that the lower intake of haem Fe appears to be compensated for by higher intake of non-haem Fe in the organic groups. In this case, the major issue concerns the lower absorption of non-haem $\mathrm{Fe}$, but this might be enhanced by co-ingested nutrients, such as vitamin C.

Finally, the small group of participants who completely avoided meat and meat products deserves special attention. Analyses of food patterns and intakes of macronutrients and micronutrients stratified for meat/meat products consumption (data not shown) revealed that the differences between the organic groups and the conventional (reference) group were especially marked among the participants who completely avoided meat and meat products, but were in line with the differences observed among the participants who consume meat and meat products.

\section{Implications}

From the general population recruitment group we can derive an estimation of the use of food of organic origin in the general population in families of childbearing age at the time when the questionnaires were collected (20002002). Our data show that a considerable part of the general population recruitment group purchased at least some products of organic origin. Furthermore, the food pattern of the group who consumed organic products in moderation ( $<50 \%$ organic group) differed significantly from the conventional (reference) group. Sometimes the differences between this organic group and the conventional one were even higher than in the other organic groups. This shows that consumption of some organic food is already associated with a markedly different food pattern, thereby suggesting that such patterns are widespread in the general population. There are several indications that consumption of food of organic origin is likely to have increased in the years after the questionnaires were 
collected. For instance, between 2005 and 2011, there was a $50 \%$ increase in organic farming in the EU, accompanied by considerable growth in sales of organic products on the EU food market, which reached $€ 19600$ million in 2010 compared with $€ 16000$ million in $2007^{(17)}$.

Until now, only a few studies have been performed on the influence of organic food on health, especially women's health during pregnancy ${ }^{(2,18)}$, and effects on the offspring $^{(3,4)}$. Our results suggest that organic food users tend to have a diet closer to dietary recommendations, i.e. possibly healthier. Therefore, when studying the impact of the type of food origin on health-related characteristics, not only the amounts of organic products should be considered, but also the food patterns and intakes must be analysed. All groups exhibited a relatively well-balanced food pattern and intake. Nevertheless, the organic groups were characterized by lower intake of products of animal origin and higher intake of products of plant origin, which according to the available evidence ${ }^{(19)}$ might be beneficial for health. Indeed, products with high levels of trans- and saturated fatty acids, including red and processed meats, have been shown to exert a negative impact on health ${ }^{(19)}$. At the same time, increased fruit and vegetable intake is associated with decreased risk of CVD, cancer and overall mortality.

Organic farming per se plays a role in the achievement of more sustainable consumption and production ${ }^{(17)}$. The observation that organic food consumers are adopting food consumption patterns that rely more on plant and less on animal products suggests additional gains in terms of the sustainability of human nutrition.

\section{Study strengths and limitations}

Strengths of the present work comprise the extensive assessment of lifestyle characteristics, the very detailed FFQ and the specific recruitment of some of the participants through 'alternative' recruitment channels. In addition, we were able to compare a wide range of users of organic products, ranging from occasional users to very consistent users.

A possible limitation is that significance testing with so many comparisons could have led to some false-positive significance tests; to limit this risk we used only tests for trends over the groups and used stringent criteria for reporting results of multivariate-adjusted analyses. Another possible limitation of the present study concerns the quantification of macronutrients and micronutrients in the various organic groups. Food composition tables generally do not account for differences in organic origin (except for specialty products, which were included in our FFQ to also cover organic and 'alternative' products), although a few observations suggest that at least some organic food products may contain different levels of several nutrients ${ }^{(20-28)}$. Since the generalizability of these differences in nutrient content remains uncertain and most of the observed differences were minor, they are, however, not likely to have strongly influenced our calculations.
Several subjective decisions have to be made while performing PCA, such as inclusion/exclusion or aggregation of food items. In the present work, we decided not to aggregate food items for two reasons: (i) a high number of food items has been associated with more precise estimates of disease risk ${ }^{(29)}$; and (ii) an aggregation-free PCA is likely to better complement the other approach that we have followed, namely the food intake characterization based on the highest (i.e. most aggregated) level of the NEVO-1 classification. Our data obtained with these two latter approaches gave comparable results.

\section{Conclusions}

Taken together, our results reveal the diversity of features - consumer characteristics, specificities from dietary patterns and macro- and micronutrient intakes that are associated with the consumption of organic food during pregnancy. Even consumers who buy only a small share of food of organic origin differed markedly from consumers who never buy organic food with respect to these features. Our results illustrate how important it is to take both consumer characteristics and information on dietary patterns and food intakes into account when interpreting possible effects of organic food on healthrelated characteristics.

\section{Acknowledgements}

Acknowledgements: Daniel Krüerke and Andreas Jäschke (Clinic Arlesheim) are gratefully acknowledged for facilitating the work on this project at the Clinic Arlesheim. The authors thank Dr H. Murray for language corrections. Financial support: The KOALA Cohort Study was supported by a grant from the Netherlands Organization for Health Research and Development (ZonMw Prevention Program 1, grant no. 2001-1-1260), the present analysis by Christophorus Foundation (Stuttgart, Germany). The sponsors had no role in the design, analysis or writing of this article. Conflict of interest: The authors do not have conflicts of interest. Authorship: A.P.S.-W.: study design, statistical analysis and drafting of manuscript. C.M.-P.: management and quality control of processing of food intake data. M.C.J.M.v.D.: processing of food intake data, advice on data analysis. P.C.D.: study design and data collection, C.T.: study design, coordination of data collection and supervision of statistical analysis. All authors reviewed the manuscript and agreed with the final version. Etbics of buman subject participation: The KOALA Birth Cohort Study is a prospective cohort study of 2834 mother-infant pairs in the Netherlands, which was approved by the Medical Ethical Committee of Maastricht University/Academic Hospital Maastricht (MUMC+). All children's parents signed for written informed consent. 


\section{Supplementary material}

To view supplementary material for this article, please visit http://dx.doi.org/10.1017/S1368980017000842

\section{References}

1. IFOAM Organics International (2008) What is organic agriculture? http://www.ifoam.bio/en/faq-organic-agriculture\# 12016 (accessed October 2016).

2. Torjusen H, Brantsaeter AL, Haugen M et al. (2014) Reduced risk of pre-eclampsia with organic vegetable consumption: results from the prospective Norwegian Mother and Child Cohort Study. BMJ Open 4, e006143.

3. Brantsaeter AL, Torjusen H, Meltzer HM et al. (2016) Organic food consumption during pregnancy and hypospadias and cryptorchidism at birth: the Norwegian Mother and Child Cohort Study (MoBa). Environ Health Perspect 124, 357-364.

4. Kummeling I, Thijs C, Huber M et al. (2008) Consumption of organic foods and risk of atopic disease during the first 2 years of life in the Netherlands. Br J Nutr 99, 598-605.

5. Baudry J, Mejean C, Alles B et al. (2015) Contribution of organic food to the diet in a large sample of French adults (the NutriNet-Santé Cohort Study). Nutrients 7, 8615-8632.

6. Kesse-Guyot E, Peneau S, Mejean C et al. (2013) Profiles of organic food consumers in a large sample of French adults: results from the Nutrinet-Santé cohort study. PLoS One $\mathbf{8}$, e76998.

7. Petersen SB, Rasmussen MA, Strom M et al. (2013) Sociodemographic characteristics and food habits of organic consumers - a study from the Danish National Birth Cohort. Public Health Nutr 16, 1810-1819.

8. Torjusen H, Brantsaeter AL, Haugen M et al. (2010) Characteristics associated with organic food consumption during pregnancy; data from a large cohort of pregnant women in Norway. BMC Public Health 10, 775.

9. van de Vijver LP \& van Vliet ME (2012) Health effects of an organic diet - consumer experiences in the Netherlands. J Sci Food Agric 92, 2923-2927.

10. Eisinger-Watzl M, Wittig F, Heuer T et al. (2015) Customers purchasing organic food - do they live healthier? Results of the German National Nutrition Survey II. Eur J Nutr Food Saf 5, 2347-5641.

11. Torjusen H, Lieblein G, Naes T et al. (2012) Food patterns and dietary quality associated with organic food consumption during pregnancy; data from a large cohort of pregnant women in Norway. BMC Public Health 12, 612.

12. Bastiaanssen JM, de Bie RA, Bastiaenen CH et al. (2005) Etiology and prognosis of pregnancy-related pelvic girdle pain; design of a longitudinal study. BMC Public Health $\mathbf{5}, 1$.

13. Kummeling I, Thijs C, Penders J et al. (2005) Etiology of atopy in infancy: the KOALA Birth Cohort Study. Pediatr Allergy Immunol 16, 679-684.

14. Grootenhuis PA, Westenbrink S, Sie CM et al. (1995) A semiquantitative food frequency questionnaire for use in epidemiologic research among the elderly: validation by comparison with dietary history. J Clin Epidemiol 48, 859-868.

15. Rijksinstituut voor Volksgezondheid en Milieu (National Institute for Public Health and the Environment) (2010) NEVO-online version 2010/2.0 (Dutch Food Composition Table based on Dutch Food Composition Database 2010). Bilthoven: RIVM.

16. Craig WJ (2010) Nutrition concerns and health effects of vegetarian diets. Nutr Clin Pract 25, 613-620.

17. European Commission (2013) Sustainable Development in the European Union. Luxembourg: Publications Office of the European Union.

18. Simões-Wüst AP, Kummeling I, Mommers M et al. (2013) Influence of alternative lifestyles on self-reported body weight and health characteristics in women. Eur J Public Health 24, 321-327.

19. Colditz GA (2015) Healthy diet in adults. UpToDate ${ }^{\circledR}[$ TO Lipman and L Park, editors]. https://www.uptodate.com/ contents/healthy-diet-in-adults (accessed January 2015).

20. Oliveira AB, Moura CF, Gomes-Filho E et al. (2013) The impact of organic farming on quality of tomatoes is associated to increased oxidative stress during fruit development. PLoS One 8, e56354.

21. Hallmann E, Lipowski J, Marszalek K et al. (2013) The seasonal variation in bioactive compounds content in juice from organic and non-organic tomatoes. Plant Foods Hum Nutr 68, 171-176.

22. Crinnion WJ (2010) Organic foods contain higher levels of certain nutrients, lower levels of pesticides, and may provide health benefits for the consumer. Altern Med Rev 15, 4-12.

23. Jahreis G, Fritsche J \& Steinhart H (1997) Conjugated linoleic acid in milk fat: high variation depending on production system. Nutr Res 17, 1479-1484.

24. Schwendel BH, Morel PC, Wester TJ et al. (2014) Fatty acid profile differs between organic and conventionally produced cow milk independent of season or milking time. J Dairy Sci 98, 1411-1425.

25. Ellis KA, Innocent G, Grove-White D et al. (2006) Comparing the fatty acid composition of organic and conventional milk. J Dairy Sci 89, 1938-1950.

26. Molkentin J \& Giesemann A (2007) Differentiation of organically and conventionally produced milk by stable isotope and fatty acid analysis. Anal Bioanal Chem 388, 297-305.

27. Srednicka-Tober D, Baranski M, Seal CJ et al. (2016) Higher PUFA and $n-3$ PUFA, conjugated linoleic acid, $\alpha$-tocopherol and iron, but lower iodine and selenium concentrations in organic milk: a systematic literature review and meta- and redundancy analyses. Br J Nutr 115, 1043-1060.

28. Mehdi Y \& Dufrasne I (2016) Selenium in cattle: a review. Molecules 21, 545.

29. McCann SE, Marshall JR, Brasure JR et al. (2001) Analysis of patterns of food intake in nutritional epidemiology: food classification in principal components analysis and the subsequent impact on estimates for endometrial cancer. Public Health Nutr 4, 989-997.

30. Nutrition Working Group of International Life Science Institute (1990) Recommended Daily Amounts of Vitamins \& Minerals in Europe - Report of Workshops organised by the Nutrition Working Group of International Life Science Institute. Nutr Abstr Rev 60A, 827-842. 